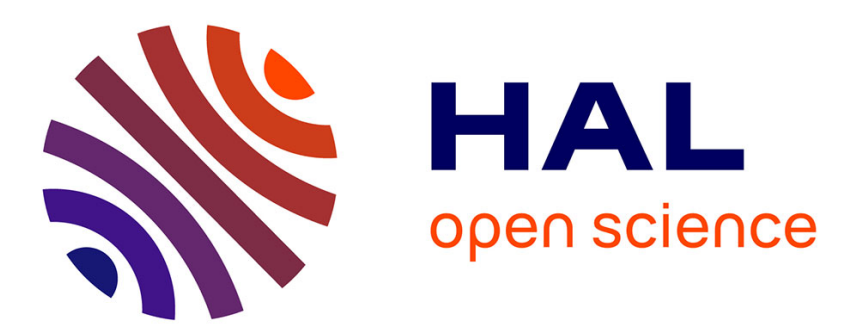

\title{
Heavy gold cluster beams production and identification
}

S. Bouneau, S. Della-Negra, J. Depauw, D. Jacquet, Y. Le Beyec, J.-P.

Mouffron, A. Novikov, M. Pautrat

\section{To cite this version:}

S. Bouneau, S. Della-Negra, J. Depauw, D. Jacquet, Y. Le Beyec, et al.. Heavy gold cluster beams production and identification. Nuclear Instruments and Methods in Physics Research Section B: Beam Interactions with Materials and Atoms, 2004, 225, pp.579-589. in2p3-00023214

HAL Id: in2p3-00023214 https://hal.in2p3.fr/in2p3-00023214

Submitted on 2 Nov 2004

HAL is a multi-disciplinary open access archive for the deposit and dissemination of scientific research documents, whether they are published or not. The documents may come from teaching and research institutions in France or abroad, or from public or private research centers.
L'archive ouverte pluridisciplinaire HAL, est destinée au dépôt et à la diffusion de documents scientifiques de niveau recherche, publiés ou non, émanant des établissements d'enseignement et de recherche français ou étrangers, des laboratoires publics ou privés. 


\title{
HEAVY GOLD CLUSTER BEAMS
}

\author{
prodation and identification
}

\author{
S. BOUNEAU, S. DELLA-NEGRA, J. DEPAUW, D. JACQUET, \\ Y. LE BEYEC, J.P. MOUFFRON, A. NOVIKOV, M. PAUTRAT \\ Institut de Physique Nucléaire, $\mathrm{IN}_{2} \mathrm{P}_{3}$-CNRS, 91406 Orsay, France
}

May 14, 2004

\begin{abstract}
Abstact
It is shown that beams of very heavy gold clusters can be produced by a liquid metal ion source (LMIS). An experimental method is described for defining the LMIS source and the Wien filter parameters that must be set to extract and select large $\mathrm{Au}_{n}$ clusters. This method is based on the acceleration of the clusters to high energy $(\mathrm{MeV})$ and on the measurement, after their passage through a thin foil, of their number of constituents and velocity. Only an average mass over charge value is obtained for a given set of source and Wien filter parameters. These parameters can then be used to select heavy $\mathrm{Au}_{n}$ cluster beams for applications at low energy (keV) in mass spectrometry.
\end{abstract}

\section{Introduction}

Beams of gold clusters, produced by a liquid metal ion source (LMIS), have been used since the early nineties to study collisions with various types of materials $[1,2,3,4,5,6]$. A large range of projectile energies was investigated, mainly in experiments on sputtering phenomena and secondary emission processes. The highest energies of $\mathrm{Au}_{n}$ clusters $(\mathrm{n}=1,13)$ were obtained by using heavy ion accelerators at Orsay and Lyon. During the course of these experiments it was observed that large size clusters could be produced with 
the gold LMIS and it became necessary, for further experiments, to know the size (i.e. the number of constituents) and the total electric charge $q$ of these entities delivered by the source.

One of the $\mathrm{Au}_{n}$ sources, including the ion optics and a Wien filter, is located at the Orsay MP Tandem terminal. A wide mass range of clusters corresponding to $\frac{n}{q}$ ratios of $5,7,9,27,40$ (selected by the Wien filter) and even nanodroplets containing up to 400 atoms are produced, accelerated by the second stage of the accelerator and sorted out by the analysing magnet. According to the parameters set on the source and the Wien filter the selection of $\frac{n}{q}$ values is obtained with a distribution of charge and mass.

In electrostatic accelerators the total particle energy depends on its charge. Therefore the measurements, after acceleration, of the cluster constituent numbers and velocities allow identification of the clusters (for a given tuning of the source). This is achieved by time-of flight measurements of the cluster atomic fragments, after passage of the incident beam through a thin Formvar foil and by measuring simultaneously, with a multianode detector [7], the number of constituents coming out of the foil. High energy clusters, accelerated at several $\mathrm{MeV}$, are thus necessary to define and verify experimentally the working parameters of the cluster ion source device. The same type of source is now used to produce large size clusters with energies below $50 \mathrm{keV}$ to investigate desorption phenomena with large projectiles as well as applications in mass spectrometry. For example, the secondary ion emission from bio-organic solids under the impact of a $\mathrm{Au}_{400}^{4+}$ beam has been studied for the first time [8].

Several sets of source parameters have been experimentally explored and this work presents the method to characterize $\mathrm{Au} u_{n}$ cluster beams and the variety of beams which can be delivered by a gold LMIS.

\section{Experimental process}

\subsection{Beam production}

\subsubsection{The ORION injector}

The gold clusters are provided by a liquid metal ion source, duplicate of the pattern developed by P. Sudraud and G. Ben Assayag [9] the specifications of which are described in references [2], [10], [11]. This simple source is made of a tungsten needle and of a reservoir containing the metal. An electric field, applied between the needlepoint and an extractor, generates a Taylor cone from which are emitted ions, clusters and nanodroplets. These are further 
accelerated by a potential of some tens of kilovolts. To obtain a low melting point, $\sim 370^{\circ} \mathrm{C}$, the reservoir contains an AuSi eutectic with 31 silicon atoms for 100 gold ones (i.e. a mass ratio of $4.4 \%$ silicon with respect to gold) [12].

The whole set-up including the ion source, two focusing lenses and a Wien filter for the mass selection of the ejected clusters is located at the accelerator terminal [10], [13]. The first cluster beams were obtained, over a large energy range, more than ten years ago. To increase the cluster beam intensities injected into the accelerator, the diameter of the extractor aperture at the source exit is set at $1.5 \mathrm{~mm}$ which leads to a $75 \mathrm{mrad}$ acceptance. In this configuration, the mass separation $\frac{m}{\Delta m}$ is between 15 and 20 . With this device it is possible to obtain ions from $\mathrm{Si}^{+}$to $\mathrm{Au}_{1000}^{+}$. Fig. 1 shows an example of mass spectrum for very heavy ions down to $\mathrm{Au}_{5}^{+}$. It shows the variation of the intensity measured by the Faraday cup, at the exit of the Wien filter, versus the voltage set on the filter plates.

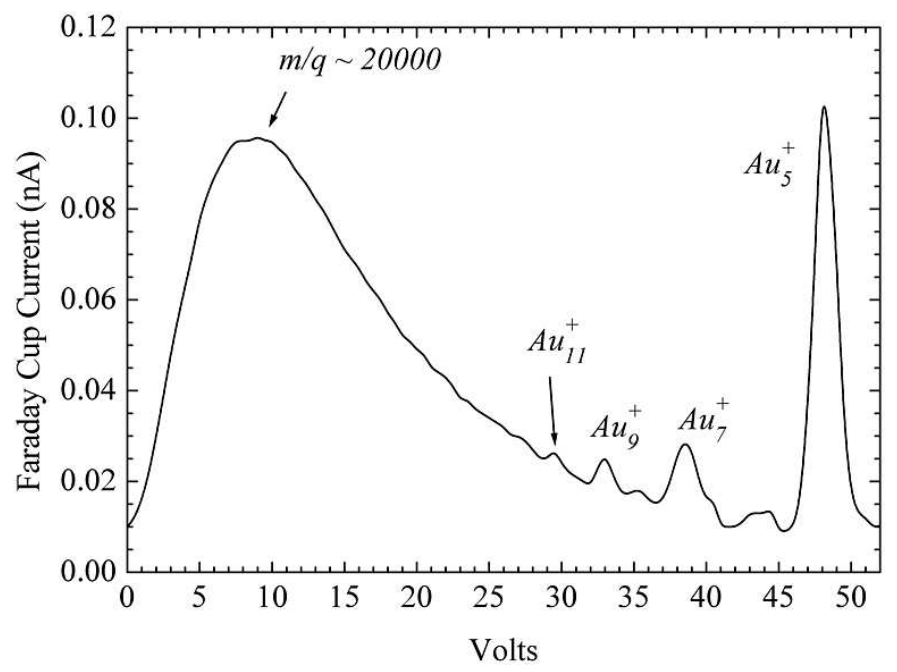

Figure 1: Current delivered by the LMIS source versus the $\mathrm{V}_{\text {Wien }}$ voltage.

Clusters containing some tens of atoms were already obtained with this source [14],[15], but in this spectrum a maximum of beam intensity is observed around a potential value of $\sim 10$ Volts corresponding to $\frac{m}{q} \sim 20000$. This ratio is deduced from the relation :

$$
\mathrm{V}_{\text {Wien }}+\delta=\mathrm{K} * \mathrm{I} * \sqrt{\frac{q}{m}}
$$

$\mathrm{V}_{\text {Wien }}($ Volts) : voltage on the electrostatic deviation plates. 
I(Amperes) : current intensity in the coils generating the magnetic field.

$\mathrm{K}$ : constant depending on the alignement of the source and Wien filter axes, experimentally adjusted.

The offset value $\delta$ depends on the beam angle at the filter inlet and a mass calibration of the Wien filter, with well known beams $\left(\mathrm{Au}^{++}, \mathrm{Au}^{+}\right.$, $\left.\mathrm{Au}_{2}^{+}, \mathrm{Au}_{5}^{+} \ldots\right)$, is advisable for each new source configuration.

Above $\frac{n}{q}=9$ it is not possible any more to separate the cluster masses since the curve of Fig. 1 becomes continuous. However, one can modify the intensity of the cluster beam as well as the maximum of its mass distribution through the adjustment of three main parameters : the voltages on the focusing lenses, the current emitted by the source and the acceleration voltage.

i) the voltages $\left(\mathrm{U}_{1}\right.$ and $\left.-\mathrm{U}_{2}\right)$ applied on the two focusing lenses allow to optimize the intensity of the selected clusters, compensating for the energy spread due to the ionization of clusters at some distance from the needle head. This energy spread increases with the cluster mass [15] and can reach $100 \mathrm{eV}$ or more. As can be seen in Fig. 2, the focusing conditions play an important role for "light" clusters but are nearly the same from $\frac{n}{q}=9$ up to 100.

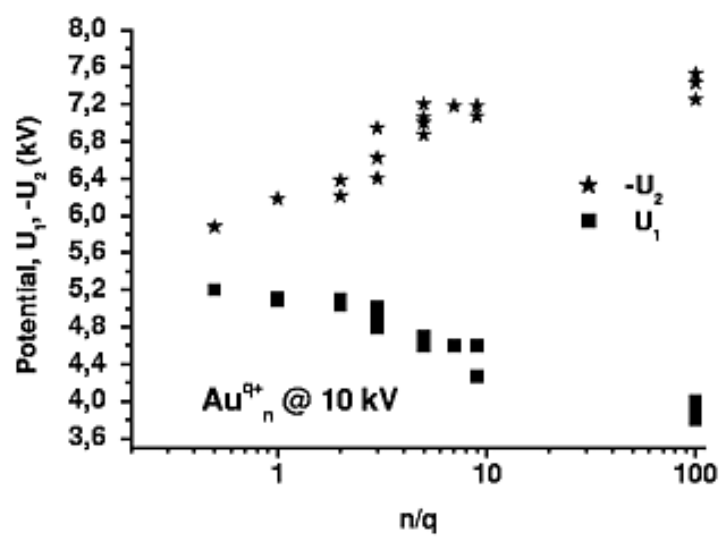

Figure 2: Optimized values of the voltages $\left(\mathrm{U}_{1},-\mathrm{U}_{2}\right)$ applied on the two focusing lenses versus the $\mathrm{n} / \mathrm{q}$ ratios of the selected clusters.

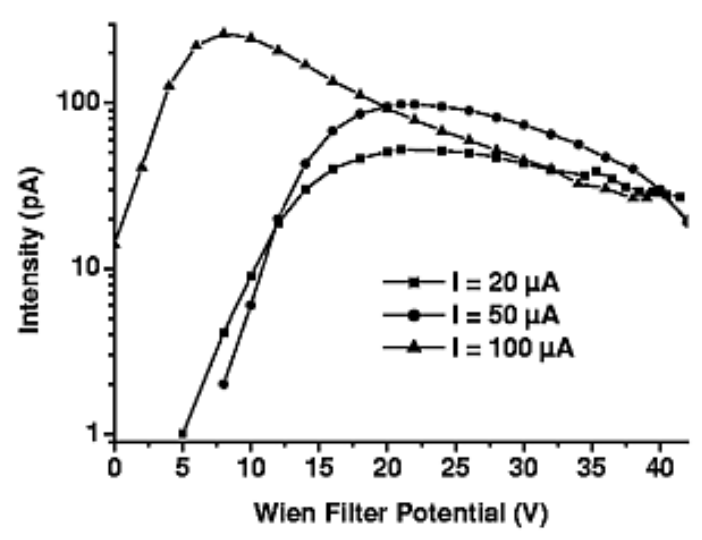

Figure 3: Intensity of the cluster beam as a function of the Wien filter potential for different source emission currents.

However, the focusing conditions can also change the shape of the beam intensity curve. The $\mathrm{Au}_{n}^{+}$peaks, for $\frac{n}{q} \geq 9$, may disappear while a "back- 
ground" increases which is not an actual background but the sum of the $\mathrm{Au}_{2 n-1}^{2+}, \mathrm{Au}_{3 n-1}^{3+}, \mathrm{Au}_{2 n+1}^{2+}, \mathrm{Au}_{3 n+1}^{3+} \ldots$ peaks.

ii) Fig. 3 shows the importance of the source emission current, as large size cluster beams, obtained for low values of the Wien filter potential (Fig. 1 ), have their highest intensity for $\mathrm{I}=100 \mu \mathrm{A}$. But a compromise must be found between the current value and the lifetime of the ion source (from 300 to 1000 hours).

iii) the acceleration voltage value is, in this case, fixed at $10 \mathrm{kV}$.

In the following, since the same $\frac{n}{q}$ ratio may be related to different cluster size and charge state values, two sets of source parameters are used for producing either low charged or highly charged clusters, which also corresponds to light or heavier ones.

\subsubsection{Acceleration and beam line}

At the Tandem terminal the selected clusters are injected, using two electrostatic mirrors, in the High Energy (HE) accelerating tube in order to reach energies from 1 to $12 . q \mathrm{MeV}$. The residual gas pressure in the tube and along the beam line is kept lower than $10^{-7} \mathrm{hPa}$ by ion and titanium vapor pumps. The beam line includes, at the machine exit, two electrostatic quadrupole lenses together with $\mathrm{X}$ and $\mathrm{Y}$ steerers, to focus and direct the beam to an analysing magnet followed by a diagnostic chamber. In the latter, by means of sliding slits associated with a microchannel plate detector (MCP) and a Faraday cup, the beam can be centered and collimated.

For $\frac{n}{q} \leq 40$, the diagnostic and collision chambers are set at a $1.29^{\circ}$ angle with respect to the beam axis and the analysing magnet eliminates the fragments of the projectile, mainly due to collisions with the residual gas. The magnetic field necessary to bend the heavier cluster trajectories would be too high and the measurements are then performed at $0^{\circ}$ without rejection of the background due to the fragments.

\subsection{Method used to characterize the ion beams}

A schematic view of the experimental set-up is given in Fig. 4. The clusters with given $\frac{n}{q}$ values are selected by the Wien filter, pre-accelerated by a $\delta U$ 
voltage in the LMIS source device and further accelerated from the terminal at a potential $U$ to the collision chamber. The total energy for a charge $q$ cluster is then :

$$
\mathrm{E}=\mathrm{q}\left[\mathrm{U}_{\text {terminal }}+\delta U_{L M I S}\right]
$$

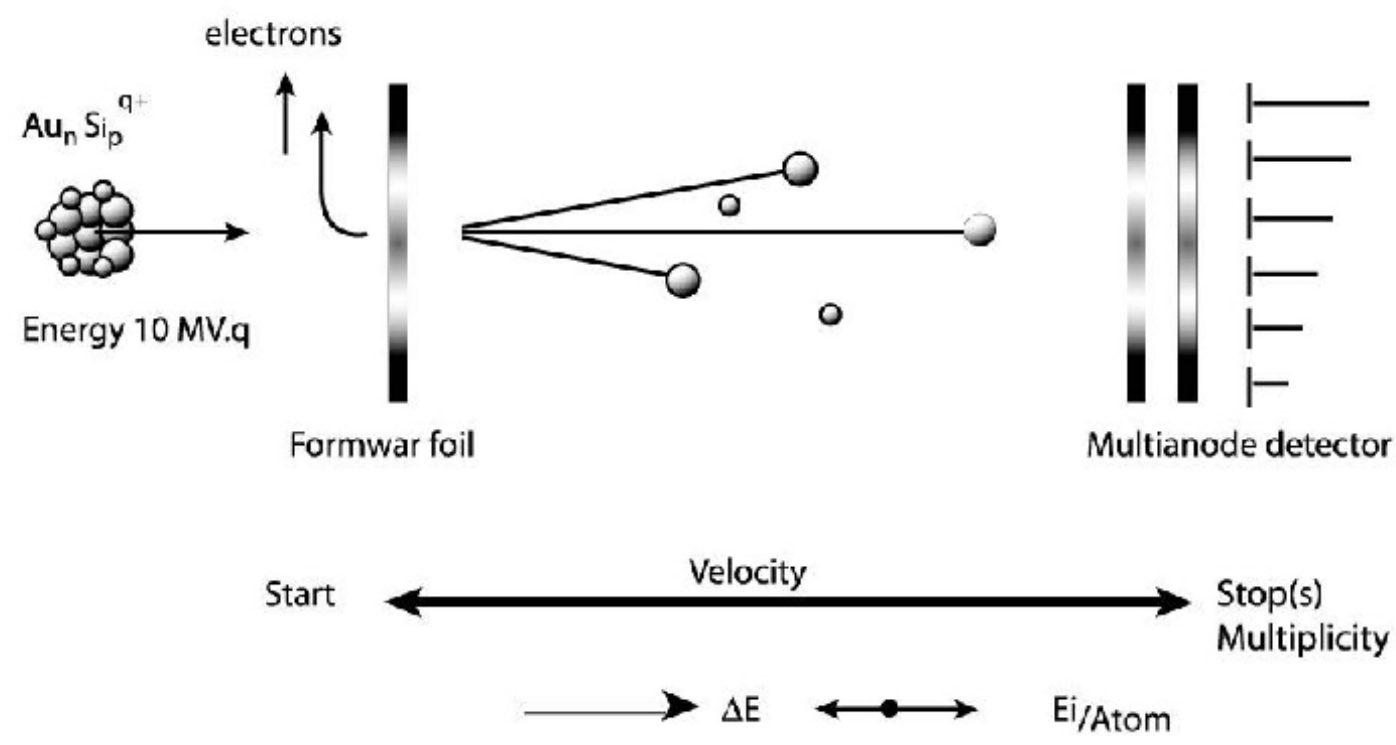

Figure 4: Schematic view of the experimental set-up.

For each charge $\mathrm{q}$ there is a distribution of $\mathrm{n}$ due to the limited mass separation power of the Wien filter. A variety of clusters, with different $q$ values and constituent numbers, are thus accelerated.

After passing through a Formvar foil perpendicular to the beam direction, a cluster dissociates into its atomic constituents and the pixelated detector records, for each incident projectile, the number of simultaneous impacts (multiplicity) on the detector. The data accumulation over a large number of events provides a multiplicity distribution with a centroid $\bar{M}$. Knowing the detector efficiency $\epsilon$ (see Appendix A), the average number of constituents $\bar{n}$ $=\frac{\bar{M}}{\epsilon}$ is obtained as well as the average charge $\bar{q}$ as $\frac{\bar{n}}{\bar{q}}=\frac{n}{q}$.

Furthermore, the time-of-flight measurement between the foil and the multianode detector gives the velocity of the Au atoms exiting the Formvar foil : 


$$
\Delta \mathrm{T}=\frac{L}{v}=\frac{L}{\sqrt{\frac{2\left(E_{1}-\Delta E\right)}{m_{A u}}}}
$$

where $\Delta \mathrm{E}$ is the calculated energy loss in the thin foil, $\mathrm{m}_{A u}$ the gold atomic mass and $\mathrm{L}$ the foil-detector distance. The time-of-flight measurement of the atomic constituents allows verification of the correct assignment of the $\frac{n}{q}$ ratio, deduced from the Wien filter parameters. Experimentally a window set on the time-of-flight peak is then used to remove the background in the measured distribution of the constituent number.

\subsection{Detection}

\subsubsection{Short description}

The electrons and negative ions emitted at the foil entrance are deflected at $90^{\circ}$ by an electrostatic mirror towards an MCP detector; the pulse delivered by the electron impact gives the start of the time-of-flight (Fig. 4). Furthermore, for $\frac{n}{q} \geq 40$, the shape of the signals due to the electrons is visualized and recorded by a digital oscilloscope, to make sure that they actually correspond to a single impact and not to several successive fragments. It is also possible to choose the $\mathrm{H}^{-}$ions as a start signal.

The projectile constituents, after the foil, are collected by a multianode detector; two different detectors of this kind have been used. The first one, described in ref. [7], is made of a set of two MCPs (Hamamatsu, $\phi=42 \mathrm{~mm}$, geometrical efficiency 0.6) and of a 256 pixel anode array $\left(1600 \mathrm{~mm}^{2}\right)$; it will be called D256 hereafter. The other smaller one (D64), has three MCPs (Hamamatsu, $\phi=28 \mathrm{~mm}$, geometrical efficiency 0.66 ) and a 64 pixel anode array $\left(400 \mathrm{~mm}^{2}\right)$.

With these detectors, located along the beam axis at $256 \mathrm{~mm}$ from the target, it is possible to measure the number of constituents and their time-of -flight. The use of the SRIM code [16] to compute the angular distributions of the constituents coming out of the foil allows calculation of the proportion reaching the detector, listed in the last column of Table 1.

Four formvar foils, with known thicknesses, are used in these experiments and the time-of-flight calibration is performed with a pure $\mathrm{Au}_{5}$ beam at a known energy.

\subsubsection{Detector efficiency}

A more complete treatment of this subject is reported in Appendix A. 
Table 1: Summary of experimental conditions

\begin{tabular}{c|c|c|c|c|c|c}
$\frac{n}{q}$ & $\begin{array}{c}\text { Energy/Au } \\
\mathrm{MeV}\end{array}$ & $\begin{array}{c}\text { Deviation } \\
\text { angle }\end{array}$ & $\begin{array}{c}\text { Formvar foil } \\
\text { thickness }(\mathrm{nm})\end{array}$ & Detector & $\begin{array}{c}\text { Ion source } \\
\text { conditions }\end{array}$ & $\begin{array}{c}\% \text { of inter- } \\
\text { cepted ions }\end{array}$ \\
\hline 5 & 2.008 & $1.29^{\circ}$ & $49 \pm 2$ & D64 & Low charge & 66 \\
7 & 1.434 & - & - & $\phi=28 \mathrm{~mm}$ & mode & 53 \\
9 & 1.115 & - & - & - & - & 44 \\
27 & 0.372 & - & - & - & - & 13 \\
5 & 1.808 & $1.29^{\circ}$ & $25 \pm 2$ & D256 & Low charge & 100 \\
40 & 0.225 & - & - & $\phi=42 \mathrm{~mm}$ & mode & 58 \\
35 & 0.287 & $0^{\circ}$ & $15 \pm 2$ & D256 & Low charge & 81 \\
120 & 0.084 & $0.03^{\circ}$ & - & - & mode & 38 \\
400 & 0.025 & $0^{\circ}$ & - & - & - & 22 \\
9 & 0.2 & $1.29^{\circ}$ & $26 \pm 2$ & D256 & High charge & 40 \\
27 & 0.2 & - & - & - & mode & - \\
40 & 0.2 & - & - & - & - & -
\end{tabular}

Two efficiencies are involved for the detector : $\epsilon_{1}$ is obtained by multiplying, for each beam, the MCPs geometrical efficiency by the percentage of intercepted ions and $\epsilon_{2}$ takes into account the pixelated structure of the anode array.

The efficiency $\epsilon_{2}$ is related to the geometrical structure of the anode and to the discriminator thresholds [7]. The current delivered by the set of MCPs, in these experiments, is always sufficient to detect the impact of gold atomic ions. The $\epsilon_{2}$ value corresponds to the detection of an impact giving a single triggering in the pixels, whereas $\left(1-\epsilon_{2}\right)$ is associated to the detection of an impact in the interpixel zone leading, in our case, to a simultaneous triggering of two adjacent pixels.

The $\epsilon_{2}$ value is close to the ratio:

$$
\epsilon_{2} \simeq \frac{\text { active geometrical surface }}{\text { total geometrical surface }}=0.74
$$

We took this value as a starting point for the multiplicity analysis, given in detail in the next section, and $\epsilon_{2}$ has been adjusted to get the best agreement with experimental results. 


\section{Experimental results and discussion}

The LMIS source delivers $\mathrm{Au}_{n} \mathrm{Si}_{p}$ clusters with a $\mathrm{p} / \mathrm{n}$ ratio of 0.1 to 0.2 , estimated from the mass spectra. In this paper, we take into account the number of gold constituents of the cluster, neglecting the silicon contribution. Through this approximation only $2 \%$ of the cluster mass is, in fact, neglected, which has no visible effect on the time-of-flight spectra. The silicon atoms are scattered, after the foil, in a solid angle about 5 times wider than that for the gold atoms and less than $10 \%$ of these reach the detector. So, the number of gold constituents measured is overestimated by $2 \%$ at the most, because of the unknown silicon atoms number.

The experimental conditions for the various beams are summarized in Table 1. Two sets of experiments are performed and compared: one for a given total energy of the projectiles and the other for the same projectile velocity.

\subsection{Time-of-flight}

For a given energy per atom (the total energy and $\frac{n}{q}$ ratio being known) one can calculate the energy loss in the Formvar foil and deduce the timeof-flight values. The measured time-of-flights values, up to $\frac{n}{q} \sim 400$, agree with the calculated ones. Furthermore, the energy spread in the foil, as well as scattering phenomena, give rise to angular and energy distributions of the exiting atoms which can be estimated with the SRIM code [16]. As a result, the calculated time-of-flight peak shapes are also shown to be in agreement with the experimental data.

An example of calculated curve is given in Fig. 5 for the $\mathrm{Au}_{5}^{+}$and $\mathrm{Au}_{9}^{+}$ projectiles, at the same $10.04 \mathrm{MeV}$ total energy. The adjustment of these curves, as well as that of the $\mathrm{Au}_{7}^{+}$one (not shown), is obtained through the convolution of the time-of-flight distribution, deduced from the SRIM energy distribution of the atomic ions, with a gaussian distribution ( $\sigma=$ $3.8 \mathrm{~ns})$ which accounts for the time-of-flight resolving power and the non homogeneity of the Formvar foil. The parameters used in the three cases are the same.

\subsection{Determination of the gold cluster masses}

Fig. 6 shows the multiplicity (i.e. the number of impacts) distributions measured, in coincidence with a time window set on the time of flight peak, 

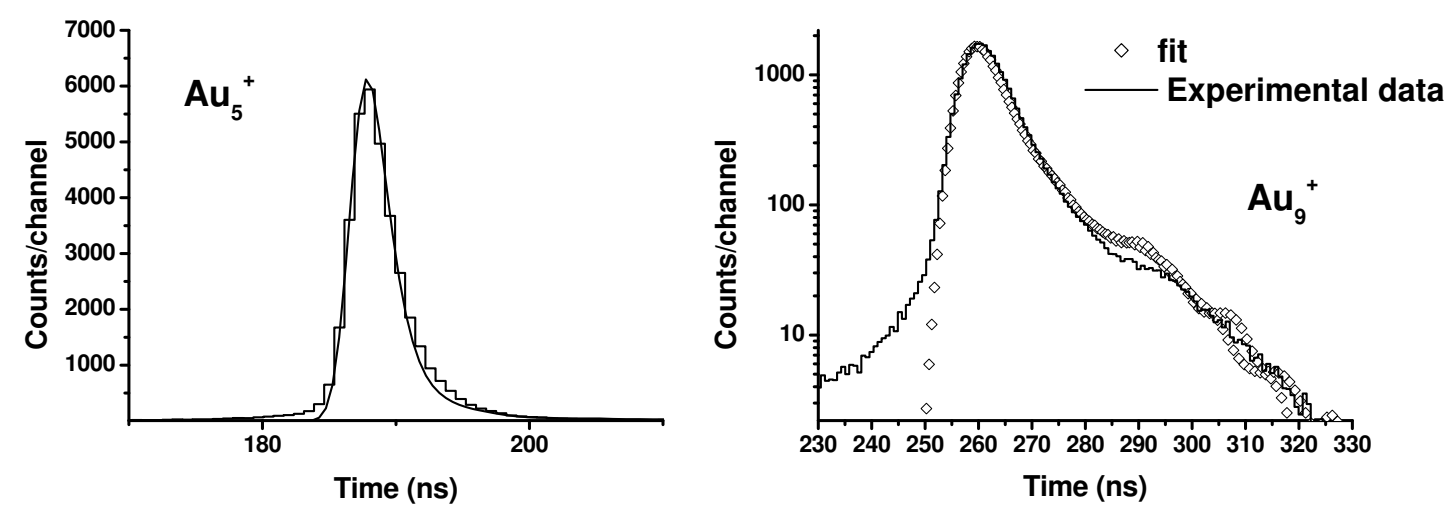

Figure 5: Experimental and calculated time-of-flight spectra for the $\mathrm{Au}_{5}^{+}$ (histogram $=$ experiment) and $\mathrm{Au}_{9}^{+}$projectiles (the time-of-flight offset value is $89 \mathrm{~ns}$ in both cases).

without magnetic selection, for voltages on the Wien filter plates corresponding to $\frac{n}{q}=35,120,400$. In Fig. 6, the mean and maximum values of the measured distribution of impact numbers increase with the $\frac{n}{q}$ selection of the Wien filter and it is possible to obtain a rough $\overline{\left(\frac{n}{q}\right)}$ estimate.

A peak of high intensity at low multiplicity, due to the previously mentionned fragmentations, disappears when the beam is selected by the H.E. analysing magnet. However, for $\frac{n}{q} \geq 40$ it is possible to use the $\mathrm{H}^{-}$signal, which emission yield exceeds $100 \%$, as a start signal to reduce the contribution of the fragmentation in the spectra. The emission of $\mathrm{H}^{-}$ions induced by fragments is, indeed, nearly negligible.

\subsubsection{Influence of the multianode structure}

There are two stages for the calculation of the number of constituents corresponding to the detection of the clusters. The probability for the MCPs to detect $\mathrm{p}$ ions among $\mathrm{n}$ follows a binomial law function of $\epsilon_{1}$ :

$$
P(p)=C_{n}^{p} \epsilon_{1}^{p}\left(1-\epsilon_{1}\right)^{n-p}
$$

The pixelated structure of the anode surface implies to take into account the $\epsilon_{2}$ efficiency as defined before. The detection probability of all the anode 


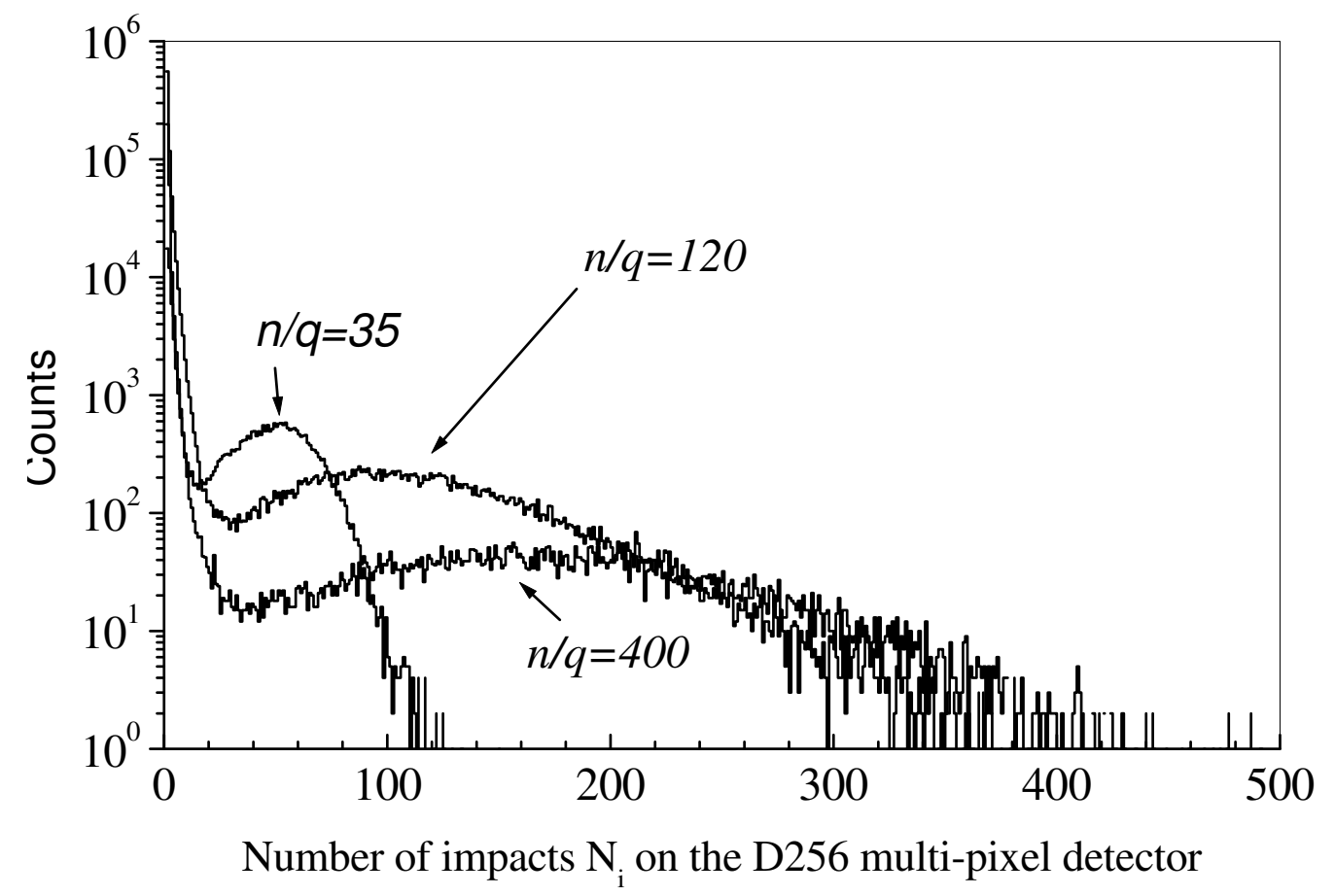

Figure 6: Number of counts versus the measured multiplicity, without magnetic field and for several Wien filter settings, in the low mass ion source conditions.

central parts obeys also a binomial law, function of $\epsilon_{2}$, and the probability of detecting $\mathrm{r}$ ions among $\mathrm{n}$ is the product of these two binomials :

$$
P(r)=C_{n}^{r} \epsilon_{1}^{r} \epsilon_{2}^{r}\left(1-\epsilon_{1} \epsilon_{2}\right)^{n-r}
$$

An ion falling in the interpixel area may be detected by the two adjoining pixels. The binomial law is the same, but the corresponding multiplicity is $\mathrm{M}=2(\mathrm{p}-\mathrm{r})$.

The variation of the detection probability in terms of the multiplicity finally assigns to each $\mathrm{P}(\mathrm{r})$ value a $\mathrm{M}=\mathrm{r}+2(\mathrm{p}-\mathrm{r})=2 \mathrm{p}-\mathrm{r}$ multiplicity. Then, the distribution of the detected ions as a function of the multiplicity reads, as before :

$$
P(M)=\sum_{p} \sum_{r} C_{n}^{p} C_{p}^{r} \epsilon_{1}^{p}\left(1-\epsilon_{1}\right)^{n-p} \epsilon_{2}^{r}\left(1-\epsilon_{2}\right)^{p-r}
$$




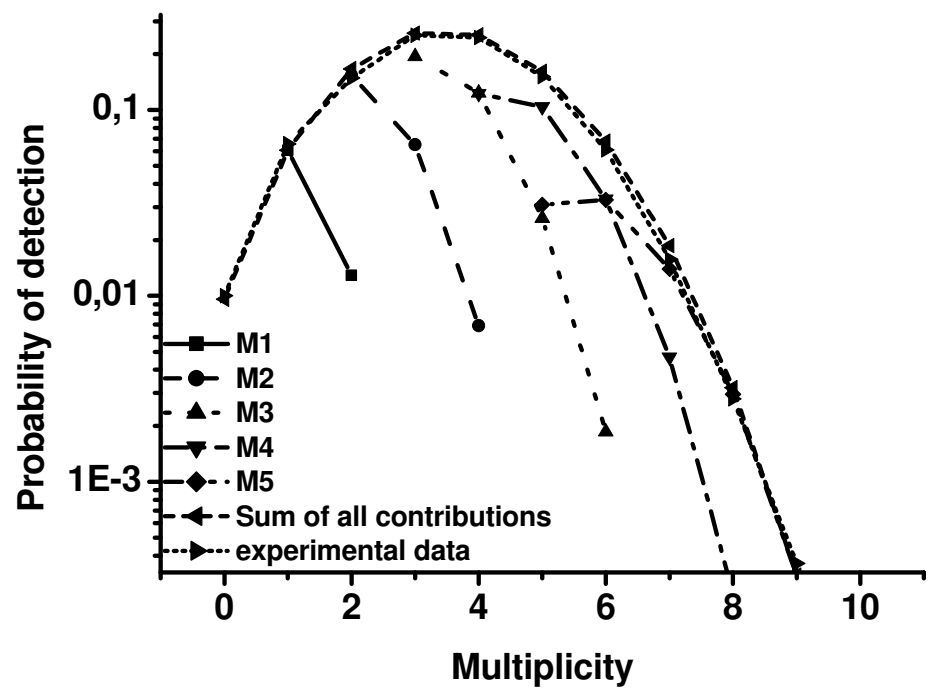

Figure 7: Calculated contributions of the $M=1$ to 5 detected ions and comparison of their sum with the experimental distribution for $\mathrm{Au}_{5}$

with $\mathrm{M}=2 \mathrm{p}-\mathrm{r}$

The experimental multiplicity, obtained with the D256 detector, for a 9 $\mathrm{MeV} \mathrm{Au}+5$ beam, is displayed in Fig. 7. The measured multiplicity values go beyond 5 because of the multiple triggering of ions falling in the interpixel zone. Taking this into account as described above, an interpixel surface leading to $18 \%$ of double hits gives a perfect fit of the measured multiplicity spectrum. The events associated to one to five impacts (marked $M_{i} i=1$ to 5) are detailed in Fig. 7.

\subsubsection{Average mass measurement}

The various multiplicity curves, for the single-charged clusters $A u_{5}, A u_{7}$, $\mathrm{Au}_{9}$, were fitted with the already determined $\epsilon_{1}$ efficiency (detection yield and solid angle calculation) and by adjusting the $\epsilon_{2}$ parameter. An $\epsilon_{2}$ value, lower than 0.74 , indicates that the surface responsible for multiplicities of 2 is larger than the interpixel surface, this effect being related to the detector gain.

For clusters with a $\frac{n}{q}$ ratio of 27 and 40 , higher charge states must be considered to fit the distribution. 
Table 2: Efficiencies of the two detectors for the studied clusters and measured average multiplicities $\bar{M}$

\begin{tabular}{|c|c|c|c|c|c|c|c|c|c|}
\hline \multirow[b]{2}{*}{$\begin{array}{c}\text { source } \\
\text { parameters }\end{array}$} & \multirow[b]{2}{*}{$n / q$} & \multicolumn{4}{|c|}{ D64 } & \multicolumn{4}{|c|}{ D256 } \\
\hline & & $\begin{array}{c}\text { E/atom } \\
(\mathrm{MeV})\end{array}$ & $\epsilon_{1}$ & $\epsilon_{2}$ & $\bar{M}$ & $\begin{array}{c}\text { E/atom } \\
(\mathrm{MeV})\end{array}$ & $\epsilon_{1}$ & $\epsilon_{2}$ & $\bar{M}$ \\
\hline \multirow{6}{*}{ Low charge } & 5 & 2 & 0.42 & 0.57 & 3.46 & \multirow[t]{4}{*}{1.8} & \multirow[t]{4}{*}{0.60} & \multirow[t]{4}{*}{0.82} & \multirow[t]{4}{*}{3.5} \\
\hline & 7 & 1.43 & 0.28 & 0.6 & 3.4 & & & & \\
\hline & 9 & 1.11 & 0.24 & 0.73 & 3.38 & & & & \\
\hline & 27 & .37 & 0.08 & 0.7 & 9.04 & & & & \\
\hline & 40 & & & & & 0.225 & 0.35 & 0.8 & 57.3 \\
\hline & 120 & & & & & 0.084 & 0.23 & 0.8 & 154 \\
\hline & 9 & & & & & 0.2 & 0.24 & 0.75 & 4.62 \\
\hline High charge & 27 & & & & & 0.2 & - & - & 30.4 \\
\hline & 40 & & & & & 0.2 & - & - & 54.7 \\
\hline
\end{tabular}

In Table 2 are listed, for the different $\frac{n}{q}$ and energy values, the $\epsilon_{1}$ and $\epsilon_{2}$ efficiencies used and the measured average multiplicity; $\epsilon_{1}$ and $\epsilon_{2}$ have been adjusted, around the values provided by the experimental conditions, to reach the best agreement between measured and calculated distributions in terms of the multiplicity. The slight variation of $\epsilon_{2}$ with the constituent number should be connected to the detector gain and to the influence of the velocity on the MCPs response under the impact of gold ions.

\subsubsection{Charge state determination}

The Fig. 8 spectra are obtained with the ion source parameters which favour the low mass cluster beams. From Fig. 8a one can see that, even in the $\frac{n}{q}=9$ case, a small proportion of $\mathrm{Au}_{18}^{++}$added to the prevailing $\mathrm{Au}_{9}^{+}$contribution is necessary to reproduce the multiplicity distribution. In Fig. $8 \mathrm{~b}$ are compared the experimental multiplicity distribution for $\frac{n}{q}=40$ and the sum of the computed contributions of the different charge states involved. A good fit of the experimental result needs to include the $\mathrm{q}=1^{+}$to $5^{+}$charge states. The contributions of the various charge states which, when summing them up, 

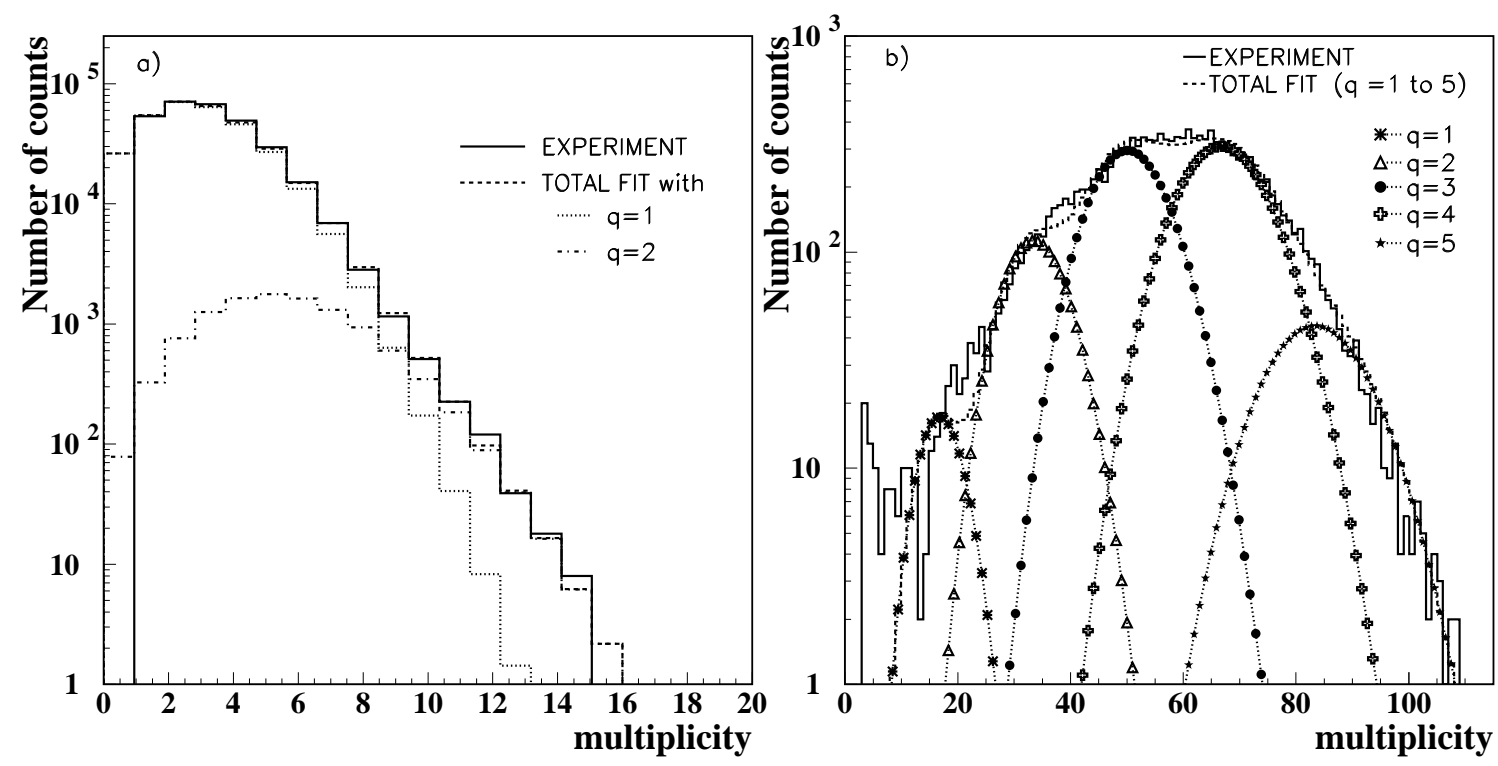

Figure 8: Comparison between the multiplicity distributions measured and computed for a) $\frac{n}{q}=9$ and b) $\frac{n}{q}=40$ taking into account several charge states.

Table 3: Percentages of the charge states involved for several $\frac{n}{q}$ values and for two ion source regimes.

\begin{tabular}{ccc|ccccccccc|c} 
& & $\mathrm{q}$ & 1 & 2 & 3 & 4 & 5 & 6 & 7 & 8 & 9 & $\bar{q}$ \\
& $\mathrm{n} / \mathrm{q}$ & & & & & & & & & & \\
\hline Low & 5 & 98.7 & 1.3 & & & & & & & & 1.01 \\
charge & 7 & 94 & 6 & & & & & & & & 1.06 \\
& 9 & 96.6 & 3.4 & & & & & & & & 1.03 \\
& 27 & 24.6 & 18.8 & 19.2 & 15.5 & 13.8 & 8.1 & & & & 3 \\
& 40 & 1 & 11 & 36 & 44 & 7 & & & & & 3.4 \\
& 120 & 3.1 & 7.4 & 16 & 20.9 & 18 & 13.4 & 10.3 & 6 & 4.7 & 4.8 \\
High & 9 & 23 & 74 & 3 & & & & & & \\
charge & 27 & 4.1 & 13.3 & 26 & 34.8 & 16.6 & 6 & & & & 1.8 \\
& 40 & 2 & 4.5 & 12 & 25 & 31 & 19.5 & 6 & & & 4.6 \\
& & & & & & & & & & & &
\end{tabular}

give the best agreement between experiment and calculation for different $\frac{n}{q}$ 
values, are listed in Table 3, for two sets of the ion source parameters. The Table 3 values (percentages and deduced mean q value) are accurate within $\pm 10 \%$.

Table 3 shows that the relative contribution of the high charge states increases with $\frac{n}{q}$, and that the ion source conditions play a crucial part. Going from the "low charge" to the "high charge" conditions, the average charge goes up by $80 \%$ for the ions with a $\frac{n}{q}=9$ ratio, and by $35 \%$ for $\frac{n}{q}$ $=40$.

\section{Conclusion}

The LMIS source delivers gold clusters from $\mathrm{Au}_{2}^{+}$to $\mathrm{Au}_{1000}^{q+}$ or more. Previous results have shown that a cluster distribution exists for $\frac{n}{q}$ ratios between 10 and 1000 (i.e. masses between 2000 and $200000 \mathrm{amu}$ ) with a maximum for $\frac{n}{q}=100$ and with a beam intensity identical to that of $\mathrm{Au}_{5}^{+}$.

The cluster ions produced by a LMIS source and selected by a Wien filter are identified for a large mass to charge range going from $\mathrm{Au}_{5}^{+}$to $\mathrm{Au}_{120 q}^{q+}$. The mass is deduced from the measurement of the constituent number of the cluster, accelerated at an energy of several MV.q and broken up by passing through a thin foil. It is thus possible to obtain the mass and charge distributions of the cluster ions. For the maximum $\frac{n}{q}$ value explored here (120), the mean charge value is 5 and the mass distribution extends from $\mathrm{Au}_{120}^{+}$to $\mathrm{Au}_{1080}^{9+}$. A correlation has been established between the mass and charge distributions and the ion source working conditions.

These experiments lead to a better knowledge of the LMIS source parameters and possibilities, in the fields of secondary emission and ion implantation in the keV energy range. Secondary ion emission rates greater than those ever observed should now be available with a nanoparticle beam. Moreover, in the near future, the use of micro beams of these heavy clusters may be considered for imaging of biological samples, with 1 to $10 \mu \mathrm{m}$ dimensions and on a $10 \mathrm{~nm}$ depth.

\section{Appendix A}

\section{Featuring of the multianode detectors.}

It is achieved following the procedure worked out in Ref. [7]. Three parameters must be determined : 
- the geometrical efficiency of the first MCP detector.

- the detection loss due to the discriminator thresholds depending on the charge collected by the anodes, i.e. on the number of electrons emitted under ion impact and on the whole detector gain.

- the efficiency of the anode array made of pixels with, in the interpixel zone, a separating grid surrounded by an insulating area to eliminate crosstalk between anodes. This configuration induces a special response of the interpixel. If the electron cloud coming out of the last MCP is in the interpixel zone, the respective positions of this cloud and of the interpixel center give rise to three possibilities:

- A single anode is hit and the incident ion is detected by one anode.

- The two anodes are hit simultaneously and the ion is recorded twice.

- The charge collected by the anodes is not sufficient and the impact is not observed.

The anode array efficiency splits up into the three probabilities :

$\mathrm{P}_{0} \quad$ no detection.

$\mathrm{P}_{1} \quad$ detection by a single anode.

$\mathrm{P}_{2} \quad$ detection by two adjoining anodes.

with the relation :

$$
\mathrm{P}_{0}+\mathrm{P}_{1}+\mathrm{P}_{2}=1
$$

The basic idea for the determination of the absolute efficiencies, or detection probabilities, is the coincidence between two detectors recording the same event. If the total number of particles is $\mathrm{N}_{T o t}$ the first detector gives :

$$
\mathrm{N}_{\text {Ref. }}=\mathrm{N}_{0}=\epsilon \cdot \mathrm{N}_{\text {Tot. }}
$$

For the second detector, which must be calibrated :

$$
\mathrm{N}_{1}=\epsilon_{1} \cdot \mathrm{N}_{T o t}
$$

and the number of coincidences is :

$$
\begin{gathered}
\mathrm{N}_{\text {coinc. }}=\epsilon . \epsilon_{1} \cdot \mathrm{N}_{\text {Tot. }} . \\
\epsilon_{1}=\frac{N_{\text {coinc. }}}{N_{0}}
\end{gathered}
$$

is the effective detection probability.

Actually, account must be taken of the detector backgrounds, $\mathrm{N}_{0}^{B}$ and $\mathrm{N}_{1}^{B}$, as well as of the correction for the data loss in the discriminators (D). The detection probability $\mathrm{P}_{d e t}$ is then written : 


$$
\begin{gathered}
\mathrm{P}_{\text {det. }}=\frac{N \text { coinc. }-N_{1}^{B}}{N_{0}-N_{0}^{B}} \cdot \frac{1}{D} \\
\epsilon_{1}=\mathrm{P}_{\text {det. }} . \mathrm{D}
\end{gathered}
$$

If a transmitting grid $\mathrm{T}$ is on the beam path, equations (7) and (8) become:

$$
\begin{gathered}
\mathrm{P}_{\text {det. }}=\frac{N \operatorname{coinc} .-N_{1}^{B}}{N_{0}-N_{0}^{B}} \cdot \frac{1}{D . T} \\
\epsilon_{1}=\mathrm{P}_{\text {det. }} . \mathrm{D} . \mathrm{T}
\end{gathered}
$$

If the detection solid angle does not cover the whole emission angle, it is necessary to add another term $\omega$ describing the average collection percentage of the detector This term is equivalent to the transmission of a grid set on the incident ions path:

$$
\epsilon_{1}=\mathrm{P}_{\text {det. }} . \text { D.T. } \omega
$$

In our detector case, with a pixelated anode array, two procedures are available :

- either a small dimension beam is used to scan the detector, which provides it's actual efficiency in each $(\mathrm{x}, \mathrm{y})$ point (the coincidence number then corresponds to an event with at least one hit anode) :

$$
\bar{\epsilon}_{1}=\frac{1}{S} \iint_{S} \epsilon(x, y) d x d y
$$

where $\mathrm{S}$ is the surface of the detector or of an anode array cell.

- or the beam covers the whole detector and the effective efficiency takes into account the anode efficiency as for a grid crossing the ions path (equations 9,10$)$.

To find out the equation (2) terms, a scan with a sharp beam tests the interpixel response. The yields associated to a single anode or to two neighbouring ones are measured in terms of the position, and the $\mathrm{P}_{1}$ and $\mathrm{P}_{2}$ probabilities of equation (2) are so determined, as well as $\mathrm{P}_{0}$ marking the lack of detection. Fig. 9a shows an example of these measurements; it presents the response of an area of the D64 detector to a $100 \mu \mathrm{m}$ x $100 \mu \mathrm{m} \mathrm{Au}_{5}^{+}$beam, with a $30 \mathrm{mV}$ discriminator threshold.

It can be seen, on this figure, that in the interpixel center (1.4 $\mathrm{mm}$ position) the multiplicity 2 yield is maximum (two active anodes), greater than the value for a single hit anode. On the other hand, the total detection yield 

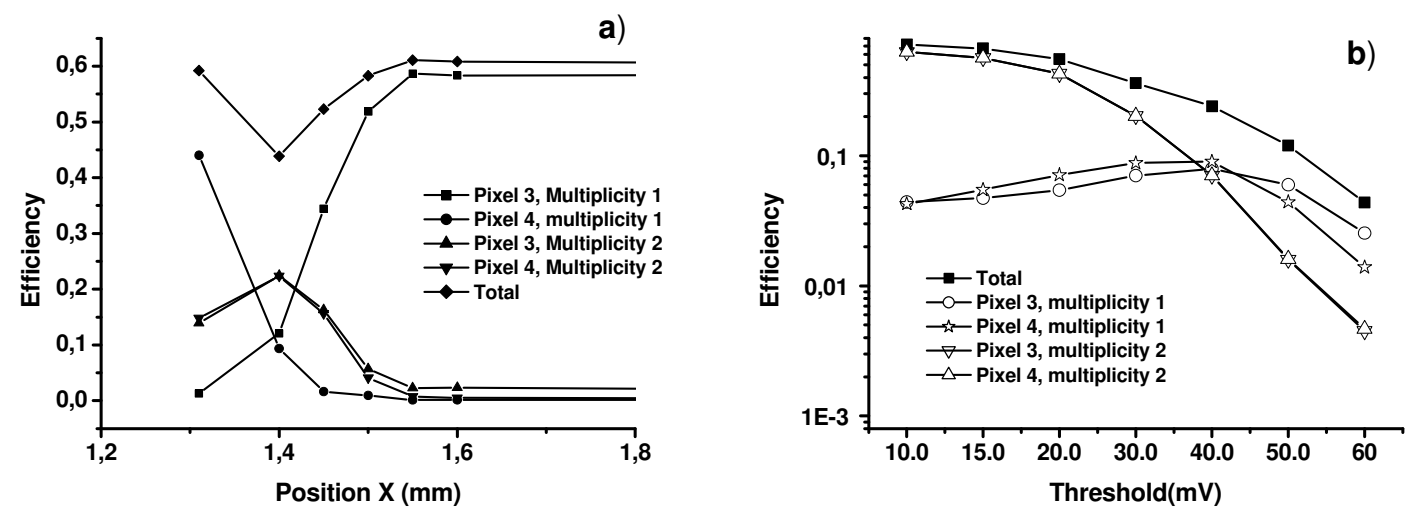

Figure 9: D64 efficiency measured a) versus the detector position, for a 30 $\mathrm{mV}$ threshold $\mathrm{b}$ ) versus the threshold in the middle of the interpixel zone.

is $26 \%$ smaller, in this position, than that obtained in the middle of the adjoining pixel. We so have indeed access to $\mathrm{P}_{0}(\mathrm{x}, \mathrm{y}), \mathrm{P}_{1}(\mathrm{x}, \mathrm{y}), \mathrm{P}_{2}(\mathrm{x}, \mathrm{y})$.

Obviously, the $\mathrm{P}_{0}, \mathrm{P}_{1}$ and $\mathrm{P}_{2}$ probabilities do vary with the charge per impact delivered by the detector, and/or with the discriminator threshold as shown in Fig. 9b where this analysis is made in the middle of the interpixel. In our case, we take into account $\mathrm{P}_{1}$ and $\mathrm{P}_{2}$, while $\mathrm{P}_{0}$ is included in $\epsilon_{1}$. The notations used in this work are :

$$
\epsilon_{2}=\mathrm{P}_{1} \text { and } 1-\epsilon_{2}=\mathrm{P}_{2}
$$

For this study, the discriminator thresholds are set at $10 \mathrm{mV}$, so that the impacts producing two electons at least are detected, and the detector gains are adjusted to obtain pulses with an average amplitude of about a hundred $\mathrm{mV}$. The electron emission from a carbon foil following a gold atom impact is of about 10 to 20 electrons, with a factor of 2 between $300 \mathrm{keV}$ and $2 \mathrm{MeV}$ [18], and a full cluster impact follows a sublinear law in $\mathrm{n}^{0.6}, \mathrm{n}$ being the constituent number [17] [18]. With these conditions for the electron emission, it is possible to neglect the $10 \mathrm{mV}$ threshold as it has no effect. We were able to verify that $\mathrm{D} \simeq 1$ and that the interpixel loss is minimum, which agrees with $\mathrm{P}_{0} \simeq 0$.

\section{References}

[1] M.G. Blain, S. Della-Negra, H. Joret, Y Le Beyec and E.A. Schweikert, Phys. Rev. Lett. 63 (1989) 1625. 
[2] M. Benguerba, A. Brunelle, S. Della-Negra, J. Depauw, M. Joret, Y. Le Beyec, M.G. Blain, E.A. Schweikert, G. Ben Assayag and P. Sudraud, Nucl. Instr. and Meth.in Phys. Res. B 62 (1991) 8.

[3] H.H. Andersen, A. Brunelle, S. Della-Negra, J. Depauw, D. Jacquet, Y. Le Beyec, J. Chaumont, and H. Bernas. Phys. Rev. Lett. 80 (1998) 5433.

[4] A. Brunelle, S. Della-Negra, J. Depauw, D. Jacquet, Y. Le Beyec, M. Pautrat, K. Baudin and H.H. Andersen, Phys. Rev. A 63 (2001) 022902.

[5] S. Bouneau, A. Brunelle, S. Della-Negra, J. Depauw, D. Jacquet, Y. Le Beyec, M. Pautrat, M. Fallavier, J.C. Poizat and H.H. Andersen, Phys. Rev. B 65 (2002) 144106.

[6] M. Fallavier, R. Kirsch, S.N. Morozov, J.C. Poizat, J.P. Thomas and N. Wehbe, Phys. Rev. B 68 (2003) 140102(R).

[7] S. Bouneau, P. Cohen, S. Della-Negra, D. Jacquet, Y. Le Beyec, J. Le Bris, M. Pautrat and R. Sellem, Rev. Sci. Instrum. 74 (2003) 57.

[8] A. Tempez, J.A. Schultz, S. Della-Negra, J. Depauw, D. Jacquet, A. Novikov, Y Le Beyec, M. Pautrat, M. Caroff, M. Ugarov, H. Bensaoula, M. Gonin, K. Fuhrer and A. Woods, Rapid Commun. Mass Spectrom. 18 (2004) 371. [9] G. Ben Assayag, Thesis, Université Paul Sabatier, Toulouse (1984).

[10] P. Attal, S. Della-Negra, D. Gardès, J.D. Larson, Y. Le Beyec, R. VienetLegué and B. Waast, Nucl. Instr. and Meth. in Phys. Res. A 328 (1993) 293.

[11] K. Boussofiane-Baudin, Thesis, Université de Paris Sud, Orsay (1993) IPNO-T-93-06

[12] Di Capua, Rend. Accad. nazion. Lincei 29 (1920) 111.

[13] B. Waast, S. Della-Negra, A. Lafoux, Nucl. Instr. and Meth. in Phys. Res. A 382 (1996) 348.

[14] P. Sudraud, C. Colliex and J. van de Walle, J. Phys. Lett. 40 (1979) L.207

[15] P.D. Prewett and G.L.R. Mair, in: Focused Ion Beams from Liquid Metal Ion sources, ed. P.S. Walsh (Research Studies Press, 1991)

[16] SRIM 2000.39, www.SRIM.org.

J.F. Ziegler, J.P. Biersack and U. Littmark, in: Stopping Powers and Ranges of Ions in Matter, ed J.F. Ziegler (Pergamon Press, New York, 1985) [17] M. Fallavier, Y. Champelovier, M. Ferrari, R. Kirsch, J.C. Poizat, J.P. Thomas, B. Canut, M. Monchanin, S.M.M. Ramos, P. Thèvenard, S. DellaNegra, J.P. Mouffron, P. Nicol, The Eur. Phys. J. D 9 (1999) 629.

[18] K. Baudin, A. Brunelle, S. Della-Negra, J. Depauw, Y. Le Beyec, E.S. Parilis, Nucl. Instr. and Meth. in Phys. Res. B 117 (1996) 47. 